\title{
Crosstalk entre osso e tecido adiposo na doença renal crônica
}

\author{
Crosstalk between bone and adipose tissue in chronic kidney disease
}

\section{Autores}

Sandra Mara Silva de Azevedo Marinho

Cristiane Moraes

Denise Mafra

Universidade Federal

Fluminense - UFF.

Data de submissão: 22/08/2011 Data de aprovação: 17/02/2012

Correspondência para: Sandra Mara Silva de Azevedo Marinho Universidade Federal

Fluminense

Estrada do Muriqui, 273

Niterói - RJ-Brasil

CEP 24322-520

E-mail:

sandramaramarinho@

hotmail.com

Suporte financeiro:

Conselho Nacional de

Desenvolvimento Científico

eTecnológico (CNPq) e

Fundação de Amparo à

Pesquisa do Estado do

Rio de Janeiro (FAPERJ).

O referido estudo foi realizado na Pós-graduação em Ciências Médicas da UFF.

Os autores declaram a inexistência de conflitos de interesse.

\section{Resumo}

Dentro do conceito de que os hormônios são regulados por um ciclo de reciprocidade, o fato de osteoblastos e adipócitos serem desenvolvidos a partir de células-tronco mesenquimais e da remodelação óssea ser regulada pela leptina, traz a ideia de possíveis participações do osso no metabolismo energético e vice-versa. Estudos recentes têm demonstrado que a diferenciação e as funções das células ósseas são reguladas pela leptina, que parece desencadear uma resposta bimodal central, via sistema nervoso simpático, e uma local, na qual a leptina agiria sobre o osso. De fato, estudos têm revelado complexa interação entre osso, tecido adiposo e cérebro; no entanto, existem poucos estudos sobre esse crosstalk em pacientes com doença renal crônica (DRC). Como tais pacientes têm tendência à diminuição da densidade mineral óssea e elevados níveis de leptina, o presente artigo apresentou uma revisão sobre o possível envolvimento entre tecido adiposo e massa óssea, em pacientes com DRC.

Palavras-chave: Densidade óssea. Tecido adiposo. Leptina. Remodelação óssea.

\section{Abstract}

Within the concept that hormones are regulated by a cycle of reciprocity, the fact that osteoblasts and adipocytes are developed from mesenchymal stem cells and that bone remodeling is regulated by leptin brings up the idea of possible bone participation in energy metabolism and vice-versa. Recent studies have shown that the differentiation and function of these bone cells are regulated by leptin, which seems to trigger a bimodal response, via sympathetic nervous system, and a local response, in which leptin acts on the bone. In fact, studies have shown complex interactions between bone, adipose tissue and brain. However, there are few studies on crosstalk in patients with chronic kidney disease (CKD). These patients have a tendency to decreased bone mineral density and high levels of leptin. Then, this article presented a review of potential involvement of adipose tissue and bone mass in patients with CKD.

Keywords: Bone density. Adipose tissue. Leptin. Bone remodeling.

\section{INTRODUÇÃO}

Pacientes com doença renal crônica (DRC) têm complexas anormalidades no metabolismo mineral e ósseo, pois a redução da função renal leva a distúrbios no metabolismo do cálcio e potássio, diminuição da ativação da vitamina $\mathrm{D}$ e aumento no hormônio da paratireoide, levando a desordens ósseas. ${ }^{1}$ A distribuição da osteoporose, entre diferentes formas de osteodistrofia renal, foi observada em estudo com pacientes sob hemodiálise, e contatou-se que a osteoporose esteve presente, tanto em estados de baixa óssea (doença óssea adinâmica e osteomalácia), quanto de alta (doença óssea com hiperparatireoide predominante e osteodistrofia urêmica mista). ${ }^{2}$ Sendo assim, esses pacientes apresentam baixa densidade mineral óssea (DMO) que é causada por um desequilíbrio na atividade de dois tipos de células, primeiramente quando a atividade dos osteoclastos, responsáveis pela reabsorção óssea, supera a atividade dos osteoblastos, responsáveis pela formação 
óssea, sendo essas duas fases, de reabsorção e formação, compreendidas como remodelação óssea. ${ }^{3}$

As fraturas ósseas são as principais complicações decorrentes da diminuição da DMO e de outras alterações, em pacientes com DRC submetidos à hemodiálise, cujo risco de incidência torna-se aumentado em decorrência da osteoporose. ${ }^{4}$

De fato, a leptina parece ter um efeito bimodal sobre o crescimento e a remodelação óssea. Assim, o risco de incidência de fraturas também se encontra em associação com a diminuição dos níveis séricos de leptina, sugerindo, dessa forma, seu papel como moduladora da massa óssea. ${ }^{5}$ No entanto, são escassos os estudos com relação ao envolvimento do tecido adiposo no metabolismo ósseo de pacientes renais crônicos. Por conseguinte, essa revisão teve, como objetivo, discutir o crosstalk existente entre tecido adiposo e tecido ósseo nos pacientes renais crônicos.

\section{As VERTENTES DA OBESIDADE NA DMO}

Apesar de ser um fator de risco para várias doenças, a obesidade era tradicionalmente vista como benéfica para a saúde óssea, devido a um efeito positivo da carga mecânica conferida pela massa corporal na formação óssea. ${ }^{6}$

Filip e Raszewski ${ }^{7}$ avaliaram a DMO através da densitometria óssea, realizada através de absorciometria de raios X de dupla energia (DEXA), em mulheres na pós-menopausa, com sobrepeso e obesidade, e constataram que a DMO se correlacionou com a massa corporal (coeficiente de correlação $-r=0,52$, $\mathrm{p}<0,05)$. Além disso, através de regressão linear múltipla, constatou-se que o índice de massa corporal (IMC) foi o maior preditor, tanto da DMO na região lombar (L1 e L2) da coluna vertebral (coeficiente de correlação $\beta-\beta=0,35, \mathrm{p}<0,01$ ), quanto da DMO do colo do fêmur $(\beta=0,42, p<0,001)$.

Pesquisadores observaram que mulheres coreanas na pós-menopausa apresentaram correlação positiva entre massa corporal e DMO. No entanto, avaliando o percentual de gordura corporal e a circunferência da cintura, eles observaram uma correlação negativa com a DMO, representando, portanto, um fator de risco para fraturas vertebrais. ${ }^{8}$

Dessa forma, estudos recentes têm mostrado que excesso de massa gorda não protege o osso e causa osteoporose e, de fato, o aumento de massa gorda está associado com baixa DMO. ${ }^{6}$

Em contraposto aos benefícios da obesidade na DMO, um estudo ${ }^{9}$ com 1.406 adultos coreanos, de ambos os gêneros, apontou que a massa magra pode ser um importante determinante de DMO e que a massa gorda somente contribui positivamente para a DMO em mulheres na pós-menopausa e homens com mais de 50 anos. Foram encontrados valores expressivos de correlação positiva entre o trocanter e a massa magra, tanto nos homens com menos de 50 anos de idade $(\beta=0,680, p=0,000)$, quanto nas mulheres em pré-menopausa $(\beta=0,540, \mathrm{p}=0,000)$.

De fato, diversos estudos têm mostrado uma correlação positiva do IMC com a DMO. ${ }^{10-12}$ Todavia, também há uma grande parcela de resultados que evidenciam efeitos nocivos da obesidade para a DMO. ${ }^{9,13-15}$

Um estudo ${ }^{13}$, que avaliou chineses e caucasianos, questionou a correlação positiva entre obesidade e massa óssea. Os autores explicam que há correlação positiva entre massa corporal e DMO quando a maior parte dessa massa corporal é composta por massa magra e quando essas variáveis são ajustadas para a idade, como já mencionado em dados de outros estudos. ${ }^{8,9}$ Dessa forma, os autores ${ }^{13}$ sugerem que a massa gorda não contribui positivamente para a DMO quando os efeitos da carga mecânica da massa corporal global são estatisticamente controlados.

Outros dois trabalhos realizados corroboram com os achados de que a massa corporal está inversamente associada com a DMO. ${ }^{14,15}$ Um deles ${ }^{15}$, avaliando indivíduos de diferentes faixas etárias, concluiu que a massa gorda é negativamente associada com a massa óssea, independentemente da idade e que, a despeito da carga mecânica, o tecido adiposo não demonstrou ser benéfico para a estrutura óssea. Somado a isso, outro estudo ${ }^{14}$, realizado com chineses de ambos os gêneros, viu a razão de chances (odds ratio - OR) para a osteoporose ampliar conforme o aumento de massa corporal. Os homens com maior massa corporal, por exemplo, apresentaram aproximadamente cinco vezes mais chances de desenvolver osteoporose, quando comparados àqueles com massa corporal inferior. As mulheres com maior adiposidade que estavam na menopausa apresentaram cerca de sete vezes mais chances de desenvolver a osteoporose, quando comparadas àquelas com menor adiposidade.

Embora existam diversas óticas acerca do papel da obesidade na DMO, parece haver uma tendência em estabelecer que a obesidade seja prejudicial à saúde óssea. ${ }^{6}$ Essa interferência no metabolismo ósseo, direta ou indiretamente, ocorre pelas citocinas derivadas dos adipócitos, como, por exemplo, a leptina, pois a obesidade está associada com significante aumento de leptina e diminuição de adiponectina. ${ }^{16,17}$ 


\section{Crosstalk entre osso E TECIDO ADIPOSO}

Em 2000, Ducy et al. ${ }^{18}$ mostraram que ratos ob/ob, deficientes em leptina, exibiam elevada massa óssea, e esse estudo promoveu fortes evidências de que a leptina usa a via central para controlar a massa óssea, pois a infusão intracerebroventricular de leptina normalizava a elevada massa óssea, causada pela deficiência de leptina.

Após ser liberada pelo tecido adiposo para a corrente sanguínea, a leptina cruza a barreira hematoencefálica ligando-se aos seus receptores hipotalâmicos, informando o estado das reservas de energia corporal, e por intermédio da ligação com os seus receptores, a leptina influencia a expressão de determinados neuropeptídeos anorexígenos e orexígenos e alguns neurônios do hipotálamo. ${ }^{19-21}$

Além da expressão de neuropeptídeos envolvidos com aumento ou diminuição da ingestão alimentar, a leptina, ao se ligar com seu receptor Ob-Rb presente nos núcleos hipotalâmicos, ativa duas diferentes vias centrais que influenciam a formação óssea. $\mathrm{Na}$ primeira, através de sinalização simpática via receptores $\beta 2$-adrenérgicos (o único receptor adrenérgico presente nos osteoblastos), a leptina diminui a formação óssea nos osteoblastos e causa aumento da reabsorção óssea nos osteoclastos por induzir os receptores ativadores do fator nuclear kappa-B ligante (Rankl). O segundo mecanismo envolve a modulação do Cocaine- and Amphetamine-Regulated Transcript (CART), um neuropettídeo hipotalâmico codificado pelo gene CARTPT, cuja expressão é aumentada pela leptina, no hipotálamo ventromedial. A diminuída expressão do Rankl pelo CART inibe a diferenciação dos osteoclastos. ${ }^{8,20,22,23}$

No osteoblasto, através da ativação simpática causada no cérebro, a leptina leva à ativação de várias vias celulares que promovem diminuição da formação óssea. Além disso, como já foi dito, a ativação do CART, contrariamente à ativação simpática, leva a diminuição da reabsorção óssea. Um terceiro mediador da leptina que age no osso é a neuromedina U (NMU) que parece também diminuir a formação óssea. ${ }^{24}$

Há uma via periférica da leptina que pode também afetar o metabolismo ósseo, pois os osteoblastos possuem receptores para a leptina o que parece fazer com que haja aumento da proliferação dos osteoblastos e inibir os osteoclastos, efeitos opostos àqueles observados pela ação da leptina via central. ${ }^{25}$ No entanto, estudos realizados sobre essa ação periférica da leptina mostram resultados inconsistentes ou inconclusivos. ${ }^{26-28}$
Experimentos genéticos mostraram que a leptina pode afetar a formação óssea, assim como o apetite, ligando-se ao seu receptor através de um mensageiro secundário, a serotonina cerebral. Em vez de agir diretamente sobre os neurônios do hipotálamo, a leptina sinaliza ao seu receptor, expresso nos neurônios serotoninérgicos presentes no tronco cerebral, e essa ligação provoca uma diminuição na produção de serotonina. ${ }^{24}$

Esse ciclo recém-identificado de realimentação entre o osso e metabolismo energético é mediado pela osteocalcina, uma proteína de $5.700 \mathrm{Da}$, produzida pelo osteoblasto ${ }^{8}$. A Figura 1, na presente revisão, traz um esquema geral da conexão entre tecido adiposo, cérebro e osso.

\section{Osteocalcina e mineralização ÓsSEA}

A osteocalcina tem sido descrita como a proteína não colagenosa mais abundante nos ossos, e, durante sua biossíntese, essa proteína sofre uma série de modificações, que incluem a conversão de três resíduos de glutamato em g-ácido carboxiglutâmico (Gla) pela g-glutamilcarboxilase dependente de vitamina K. ${ }^{28,29}$ Essa g-carboxilação é essencial para que a proteína tenha uma alta afinidade com íons minerais e permite que a osteocalcina atraia íons de cálcio e incorpore-os em cristais de hidroxiapatita, os quais compõem, aproximadamente, $70 \%$ dos ossos. No entanto, nem todos os três resíduos de ácido glutâmico na osteocalcina

Figura 1. Esquema hipotético do crosstalk entre tecido adiposo e osso.

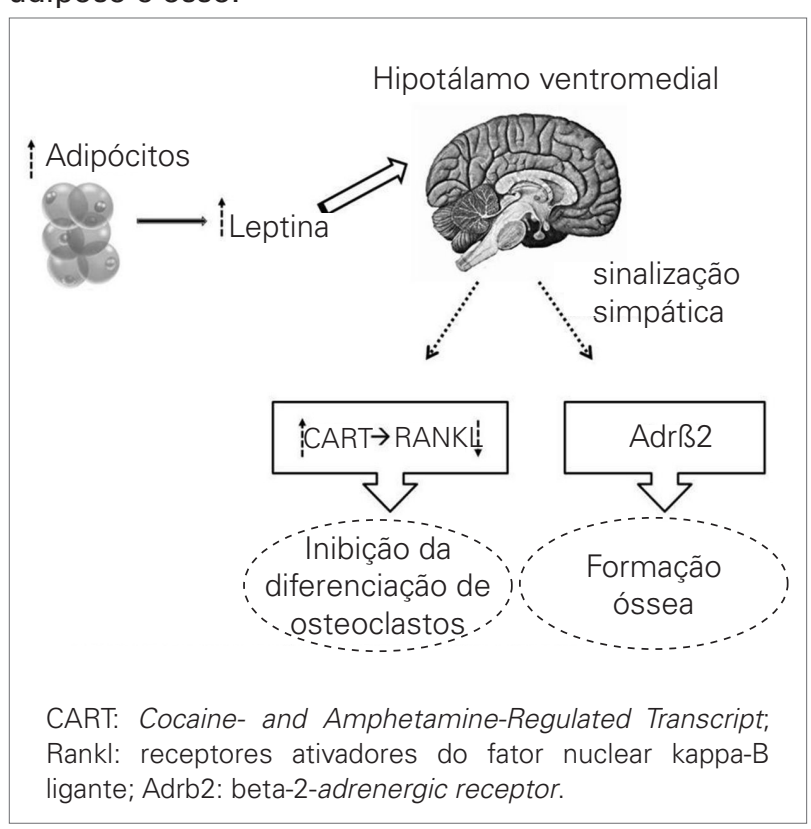


são totalmente carboxilados e incorporados aos cristais de hidroxiapatita e, o grau de carboxilação pode variar. Assim sendo, o status da vitamina $\mathrm{K}$ é especialmente importante, porque reduz a liberação de osteocalcina na circulação geral, enquanto aumenta a calcificação do osso. ${ }^{30}$ Dessa forma, a concentração plasmática de osteocalcina é um marcador clínico importante para o diagnóstico da atividade dos osteoblastos no osso. ${ }^{27}$

\section{Crosstalk entre tecido adiposo e Ósseo em PACIENTES COM DRC}

Há mais de uma década, já foi constatado que pacientes com DRC apresentam níveis séricos de leptina três vezes maiores do que indivíduos saudáveis e, somado a isso, foi também demonstrado que a leptina sérica é um excelente marcador de gordura corporal nesses pacientes. ${ }^{31}$

A DMO diminui com a deterioração da função renal em pacientes renais crônicos na pré-diálise, e a osteocalcina é um marcador clinicamente útil quando associado com a diminuição da DMO. Foi observado em pacientes renais crônicos pré-dialisados uma diminuição significativa na DMO do antebraço, no período de um ano $(\mathrm{p}<0,001)$, e teve relação com o clearance de creatinina $(\mathrm{r}=0,375, \mathrm{p}<0,01)$ e com os níveis de vitamina $\mathrm{D}$ ativa $(\mathrm{r}=0,434, \mathrm{p}<0,005)$. Em contrapartida, a osteocalcina sérica se correlacionou negativamente com as mudanças anuais da DMO $(\mathrm{r}=0,276, \mathrm{p}<0,05) .{ }^{32}$

Yilmaz et al. ${ }^{33}$ estudaram a relação entre leptina e DMO, em pacientes sob hemodiálise, e observaram correlação negativa entre os níveis de leptina e DMO da parte distal do radio $(\mathrm{r}=-0,250, \mathrm{p}=0,03)$, mesmo quando ajustado segundo o IMC $(r=-0,247$, $\mathrm{p}=0,03)$. Outro trabalho ${ }^{34}$ avaliou a correlação negativa entre leptina e DMO na região da cabeça do fêmur, em pacientes de hemodiálise e diálise peritoneal $(\mathrm{r}=-0,23, \mathrm{p}<0,05)$.

A DMO do calcâneo de 221 homens japoneses sob hemodiálise foi inversamente proporcional às concentrações séricas de leptina. Encontrou-se também uma correlação inversa entre as concentrações de leptina e o pró-peptídeo carboxiterminal do pró-colágeno tipo I (PICP), um marcador bioquímico da formação óssea. Tais dados conduzem à conclusão de que um aumento na concentração de leptina sérica pode reduzir a formação óssea e, por conseguinte, a DMO em homens adultos. ${ }^{35}$

Além da realidade já conhecida de que pacientes com DRC apresentam níveis elevados de leptina, o dimorfismo de gênero nos níveis séricos de leptina desses pacientes foi confirmado em estudo realizado com pacientes sob hemodiálise, assim também, como o fato de que esses níveis eram duas vezes mais altos nas mulheres, do que nos homens. ${ }^{34}$ Possivelmente, mesmo na ausência de obesidade, o aumento nos níveis de leptina, nesses pacientes, seja devido a uma diminuição da filtração renal, visto que, a eliminação da leptina ocorre principalmente através do rim. ${ }^{36}$ Apesar de escassos trabalhos na área de nefrologia, sugere-se o relevante papel da leptina na remodelação óssea na osteodistrofia renal.

\section{Conclusão}

Pacientes com DRC apresentam diminuição da massa óssea, o que também aumenta o risco de fraturas. Estudos recentes têm mostrado um papel modulador da leptina sobre massa mineral óssea mediado pela osteocalcina, que é produzida pelos osteoblastos. Dessa forma, torna-se plausível a hipótese de que o aumento dos níveis de leptina nos pacientes renais crônicos pode acarretar, via sistema nervoso simpático, à diminuição de osteocalcina no osso e, consequentemente, à menor densidade óssea.

\section{REFERÊNCIAS}

1. Jiang JQ, Lin S, Xu PC, Zheng DF, Jia JY. Serum osteoprotegerin measurement for early diagnosis of chronic kidney disease-mineral and bone disorder. Nephrology 2011;16:588-94.

2. Barreto FC, Barreto DV, Moyses RMA, et al. Osteoporosis in hemodialysis patients revisited by bone histomorphometry: A new insight into an old problem. Kidney Int 2006;69:1852-7.

3. Teitelbaum SL. Bone resorption by osteoclasts. Science 2000;289:1504-8.

4. Ha SK, Park CH, Seo JK, et al. Studies on bone mineral density in patients with chronic renal failure. Yonsei Med J 1996;37:350-6.

5. Doumouchtsis KK, Kostakis AI, Doumouchtsis SK, et al. Associations between osteoprotegerin and femoral neck BMD in hemodialysis patients. J Bone Miner Metab 2008;26:66-72.

6. Cao JJ. Effects of obesity on bone metabolism. J Orthop Surg Res 2011;6:30. doi:10.1186/1749-799X-6-30.

7. Filip R, Raszewski G. Bone mineral density and bone turnover in relation to serum leptin, alphaketoglutarate and sex steroids in overweight and obese postmenopausal women. Clinical Endocrinol (Oxf) 2009;70:214-20.

8. Kim KC, Shin MH, Lee SY, Im JA, Lee DC. Relation between obesity and bone mineral density and vertebral fractures in korean postmenopausal women. Yonsei Med J 2010;51:857-63.

9. Cui LH, Shin MH, Kweon SS, et al. Relative contribution of body composition to bone mineral 
density at different sites in men and women of South Korea. J Bone Miner Metab 2007;25:165-71.

10. Ravn P, Cizza G, Bjarnason NH, et al. Low body mass index is an important risk factor for low bone mass and increased bone loss in early postmenopausal women. Early Postmenopausal Intervention Cohort (EPIC) study group. J Bone Miner Res 1999;14:1622-7.

11. Felson DT, Zhang Y, Hannan MT, Anderson JJ. Effects of weight and body mass index on bone mineral density in men and women: the Framingham study. J Bone Miner Res 1993;8:567-73.

12. Reid IR. Relationships among body mass, its components, and bone. Bone 2002;31:547-55.

13. Zhao LJ, Liu YJ, Liu PY, Hamilton J, Recker RR, Deng HW. Relationship of obesity with osteoporosis. J Clin Endocrinol Metab 2007;92:1640-6.

14. Hsu YH, Venners SA, Terwedow HA, et al. Relation of body composition, fat mass, and serum lipids to osteoporotic fractures and bone mineral density in Chinese men and women. Am J Clin Nutr 2006;83:146-54.

15. Janicka A, Wren TA, Sanchez MM, et al. Fat mass is not beneficial to bone in adolescents and young adults. J Clin Endocrinol Metab 2007;92:143-7.

16. van Dielen FM, van't Veer C, Schols AM, Soeters PB, Buurman WA, Greve JW. Increased leptin concentrations correlate with increased concentrations of inflammatory markers in morbidly obese individuals. Int J Obes Relat Metab Disord 2001;25:1759-66.

17. Canavan B, Salem RO, Schurgin S, et al. Effects of physiological leptin administration on markers of inflammation, platelet activation, and platelet aggregation during caloric deprivation. J Clin Endocrinol Metab 2005;90:5779-85.

18. Ducy P, Amling M, Takeda S, et al. Leptin inhibits bone formation through a hypothalamic relay: a central control of bone mass. Cell 2000;10:197-207.

19. Mantzoros CS, Flier JS, Rogol AD. A longitudinal assessment of hormonal and physical alterations during normal puberty in boys. V. Rising leptin levels may signal the onset of puberty. J Clin Endocrinol Metab 1997;82:1066-70.

20. Takeda S, Elefteriou F, Levasseur R, et al. Leptin regulates bone formation via the sympathetic nervous system. Cell 2002;111:305-17.

21. Sahu A. Leptin signaling in the hypothalamus: emphasis on energy homeostasis and leptin resistance. Front Neuroendocrinol 2004;24:225-53.
22. Elefteriou F, Takeda S, Ebihara K, Magre J, Patano N, Kim CA. Serum leptin level is a regulator of bone mass. Proc Natl Acad Sci USA 2004;101:3258-63.

23. Thomas T, Gori F, Khosla S, Jensen MD, Burguera B, Riggs BL. Leptin acts on human marrow stromal cells to enhance differentiation to osteoblasts and to inhibit differentiation to adipocytes. Endocrinol 1999;140:1630-8.

24. Wei J, Ducy P. Co-dependence of bone and energy metabolisms. Arch Biochem Biophys 2010;503:35-40.

25. Kawai M, Sousa KM, MacDougald OA, Rosen CJ. The many facets of PPARgamma: novel insights for the skeleton. Am J Physiol Endocrinol Metab 2010;299:E3-9.

26. Ducy P, Desbois C, Boyce B, et al. Increased bone formation in osteocalcin-deficient mice. Nature 1996;382:448-52.

27. Shearer MJ. Vitamin K. Lancet 1995;345:229-34.

28. Houben RJTJ, Jin D, Stafford DW, et al. Osteocalcin binds tightly to the gamma-glutamyilcarboxilase at a site distinct from that of the other known vitamin K-dependent proteins. Biochem J 1999;341:265-9.

29. Wolf G. Function of the bone protein osteocalcin: Definitive evidence. Nut Rev 1996;54:332-3.

30. Bugel S. Vitamin K and bone health in adult humans. Vitam Horm 2008;78:393-416.

31. Heimbürger O, Lönnqvist F, Danielsson A, Nordenstrom J, Stenvinkel P. Serum immunoreactive leptin concentration and its relation to the body fat in chronic renal failure. J Am Soc Nephrol 1997;8:1423-30.

32. Obatake N, Ishimura E, Tsuchida T, et al. Annual change in bone mineral density in predialysis patients with chronic renal failure: significance of a decrease in serum 1,25-dihydroxy-vitamin D. J Bone Miner Metab 2007;25:74-9.

33. Yilmaz A, Nur N, Turgut B. Leptin and bone mineral density in haemodialysis patients. Ann Acad Med Singapore 2009;38:374-7.

34. Małyszko J, Małyszko JS, Bondyra Z, Wołczyński S, Łebkowska U, Myśliwiec M. Bone mineral density and bone metabolism are not related to leptin in hemodialyzed and peritoneally dialyzed uremic patients. Med Sci Monit 2004;10:115-9.

35. Sato M, Takeda N, Sarui H, et al. Association between serum leptin concentrations and bone mineral density, and biochemical markers of bone turnover in adult men. J Clin Endocrinol Metab 2001;86:5273-6.

36. Meyer C, Robson D, Rackovsky N, Nadkarni V, Gerich J. Role of the kidney in human leptin metabolism. Am J Physiol 1997;273:E903-7. 